\title{
Síntesis de Resinas Alquídicas a partir de Aceites de Higuerilla, de Palma y de Fritura, Mezclados con Aceite de Soja
}

\author{
Fernando Cardeño, Luis A. Rios, Jhon F. Cardona y David Ocampo \\ Universidad de Antioquia, Sede de Investigación Universitaria, cra. 53 \# 61 - 30, Medellín-Colombia. \\ (e-mail: efecl797@yahoo.com; lariospfa@gmail.com; fredycardona@gmail.com; inso99@hotmail.com )
}

Recibido Mar. 12, 2013; Aceptado Abr. 18, 2013; Versión final recibida Abr. 29, 2013

\begin{abstract}
Resumen
Se presentan los resultados obtenidos del uso de mezclas de aceites crudos de higuerilla, de palma y de fritura con aceite de soja refinado, blanqueado y desodorizado (RBD) en la síntesis de resinas alquídicas. Se emplearon las técnicas de caracterización índice de iodo, índice de acidez, índice de saponificación, humedad Karl Fisher, índice de hidroxilo, cromatografía gaseosa, transformación de Fourier de la radiación infrarroja (FTIR) y análisis termogravimétrico. Los resultados indican que las mezclas con aceite de soja que contienen 30,50 y $70 \%$ en masa de los aceites mencionados permiten obtener resinas alquídicas que cumplen con los criterios de calidad requeridos para el desarrollo de productos terminados, tales como esmaltes, lacas y selladores para madera. Estos resultados se constituyen en una alternativa tecnológica y económicamente factible para la reducción de costos mediante la incorporación de estos aceites de bajo valor dentro de la industria de resinas poliméricas tipo alquídicas o de poliéster insaturado.
\end{abstract}

Palabras clave: resinas alquídicas, aceite de palma, aceite de higuerilla, aceite de fritura, aceite de soja

\section{Synthesis of Alkyd Resins from Castor Oil, from Palm Oil and from Frying Oils, Mixed with Soybean Oil}

\begin{abstract}
Results on the synthesis of alkyd resins from mixtures of castor oil, palm oil and frying oils with refined, bleached and deodorized (RBD) soybean oil are presented. Iodine value, acidity number, saponification number, moisture content, hydroxyl number, gas chromatography, Fourier transform infrared spectroscopy (FTIR) and thermogravimetric analysis were used to characterize raw materials and products. It was found that mixtures containing 30,50 and $70 \%$ by mass of these oils, balancing with RBD soybean oil, allow obtaining alkyd resins that comply with the quality criteria required for the development of finished products such as sealants and wood coatings. These results represent a technologically and economically feasible alternative for cost reduction by incorporating these crude oils in industries that produce polymeric resins or unsaturated polyester alkyd resins
\end{abstract}




\section{INTRODUCCIÓN}

Las resinas alquídicas son las más importantes resinas sintéticas pues su volumen total utilizado en recubrimientos de superficies supera ampliamente a las demás. Se usan en casi todos los tipos de recubrimientos de superficies, pinturas, esmaltes, lacas y barnices. Las principales materias primas para su producción son aceites refinados y/o ácidos grasos altamente insaturados como el aceite de soja y el aceite de linaza, la mayoría importados para la producción de resinas alquídicas en Colombia. La producción nacional de aceites se basa en aceites poco insaturados como el aceite de palma, que no cumplen los estándares para fabricación de resinas, sin embargo podrían mezclarse parcialmente, previa modificación química, con insaturados (como por ejemplo aceites de soja) sin afectar la calidad de los productos obtenidos (Isaam, et al 2009). Sumado a esto, otros tipos de aceites como el de higuerilla, que es un aceite con propiedades relativamente importantes en cuanto a su composición en ácidos grasos tan especial (con un alto contenido de ácido ricinoleico), y la posible reutilización de aceites usados comestibles, son alternativas importantes a explotar para la generación de productos de interés comercial a partir de materias primas autóctonas de más bajo costo. (Wallace, 1978; Bakker, et al 2004; Jinkyung, et al 2007)

Como otra alternativa para reducir los costos y el impacto ambiental se ha investigado la obtención de resinas alquídicas utilizando PET (poli etilen tereftalato) reciclado, el cual se genera en una gran cantidad a nivel mundial. El uso del PET ha permitido producción de resinas alquídicas en emulsión, buscando la disminución de las emisiones de compuestos orgánicos volátiles, principalmente en países con alto desarrollo industrial en la comunidad Europea en general. (Karayannidis, et al 2005; Torlakoglu, et al 2009; Dullius, et al 2006; Murillo, et al 2011)

Hoy en día, la mayoría de los aceites de fritura se vierten en el sistema de alcantarillado de las ciudades. Esta práctica contribuye a la contaminación de ríos, lagos, mares y aguas subterráneas, lo cual es muy perjudicial para el medio ambiente y la salud humana (Lapuerta, et al 2008). Estos aceites podrían ser una materia prima con muy buenas perspectivas para las industrias de los biocombustibles y los polímeros, ya que es una materia prima más barata y se evitan los costos de eliminación como residuo. Estudios realizados en la Unión Europea muestran que se recolectan 400.000 toneladas por año de aceites de fritura o aceites usados y se piensa que puede llegar a las 700.000 toneladas por año (Lafont, et al 2011; Quiñonez, et al 2003).

La palma africana es considerada un cultivo de alto rendimiento, no solo por la cantidad de aceite que su fruto produce por hectárea sino también por la variedad de productos que del mismo y de otras partes de la planta se generan y por su utilización (actual o esperada) en la industria. En condiciones ideales, los cultivos pueden producir hasta 20 toneladas de racimos por hectárea y por año, de los cuales se puede extraer hasta el $25 \%$ de aceite de palma (cinco toneladas) y el $5 \%$ de aceite de palmiste (una tonelada). Esta cantidad es mayor que la cantidad producida por cualquier otra fuente de aceite vegetal. Algunas fuentes citan además una posible utilidad de la palma africana como sumidero de fuente de carbono y consecuentemente su posible empleo para atenuar la emisión de gases del efecto invernadero.

El aceite de higuerilla se usa ampliamente en la industria oleoquímica. Se usa para la producción de lubricantes, bases para tintas y barnices así como para la producción de adhesivos y polímeros. Este aceite ha sido considerado como una buena materia prima para la industria oleoquímica por que no se usa para alimentos y porque no requiere condiciones agrícolas exigentes para su cultivo y porque la productividad de las plantas de higuerilla es muy alta en los países tropicales. El aceite de higuerilla es químicamente muy diferente a los otros aceites considerados en este estudio, porque tiene grupos hidroxilo $(-\mathrm{OH})$ pegados a la cadena hidrocarbonada. Estos grupos hidroxilo le confiere propiedades fisicoquímicas muy diferentes e interesantes (Zuleta et al 2012).

La posible utilización del aceite de palma, de higuerilla y de aceites recuperados contribuiría a disminuir las importaciones de insumos para la fabricación de resinas alquídicas de grado comercial en Colombia, con lo cual las múltiples empresas que en nuestro país se encargan de comercializar o de procesar resinas alquídicas para su posterior aplicación en pinturas y recubrimientos se harían más competitivas frente a los retos que imponen los tratados comerciales entre los países latinoamericanos y a nivel mundial. (Hlaing, et al 2008).

Las resinas alquídicas son materiales poliméricos derivados de la reacción de polioles y poliácidos, y modificados con aceites y ácidos grasos naturales (principalmente insaturados) o sintéticos que se 
caracterizan por su secado oxidativo a temperatura ambiente en presencia de catalizadores (Panda, 2010). En general, en la elaboración de recubrimientos, las resinas alquídicas se mezclan con frecuencia con otros productos tales como las amino-resinas eterificadas (resinas derivadas de la reacción entre el formol, el isobutanol y la urea) o son modificadas con resinas poliuretánicas, poliamidas, siliconas, monómeros acrílicos y estirénicos o reducibles en agua por emulsificación para mejorar su velocidad de curado, la dureza y el aspecto, generándose productos de bajo costo, fácil aplicación y buen desempeño (Marrion, 2004)

En el presente artículo, se evalúa la sustitución total o parcial del aceite de soja por diferentes aceites crudos y usados de bajo costo, en la elaboración de las resinas alquídicas. Se determinó el efecto de dicha sustitución en las propiedades finales de la resina polimérica. Los aceites de bajo costo evaluados fueron el de higuerilla, palma y el aceite de fritura. Se buscó la obtención de resinas alquídicas que cumplan con los estándares de calidad para Colombia (NTC 1651).

\section{MATERIALES Y MÉTODOS}

\section{Reactivos}

Se emplearon como aceites para la reacción de glicerólisis: palma crudo, higuerilla crudo, residual de fritura, y soja refinado; los polioles empleados fueron: Pentaeritritol (BellChem), etilenglicol (Merk) y glicerina comercial grado USP; se emplearon Anhídridos (Panreac) ftálico y maleico, como fuente de poliácidos; como catalizador para la reacción de glicerólisis se empleó hidróxido de sodio (Merk)

\section{Equipos}

Para analizar las materias primas y los productos se emplearon: Un equipo infrarrojo (Shimadzu Prestige 21), un cromatógrafo de gases (Agilent 7890), un analizador termogravimétrico (LINSEIS STA Platinum Series), un medidor de brillo (BYK Micro-Tri-gloss 1088865), un titulador automático (Titrino 848Plus, Metrohm), un colorímetro Gárdner (BYK TKD8-02) y un viscosímetro Gárdner (BYK ,ASTM D1725)

\section{Adecuación y caracterización de aceites}

El aceite crudo de higuerilla fue sometido a un proceso de desgomado a $80^{\circ} \mathrm{C}$ con agua acidulada con $\mathrm{H}_{3} \mathrm{PO}_{4}$, con una etapa posterior de neutralización con solución alcalina de $\mathrm{NaOH}$, seguida de centrifugación a 8000 rpm por 30 min y lavado con agua y posterior secado por evaporación (Sevim, et al 2005). El aceite de palma crudo fue sometido a un proceso de neutralización de la acidez libre empleando solución alcalina de $\mathrm{NaOH}$ (hasta $\mathrm{pH}$ 7-8), el aceite de fritura fue sometido a un proceso de desecación con agitación constante empleando $\mathrm{NaCl}$ y $\mathrm{Na}_{2} \mathrm{SO}_{4}\left(0.5 \%\right.$ en peso del aceite) a una temperatura entre $75-80^{\circ} \mathrm{C}$ durante una hora con posterior decoloración con carbón activado, decantación y filtración de las impurezas sólidas usando carbón activado como material absorbente y finalmente neutralizando la acidez remante. Los productos obtenidos fueron caracterizados de acuerdo a la Tabla 3.

\section{Preparación y caracterización de la resinas alquídicas}

\section{Etapa de glicerólisis}

La preparación de la resina se realizó una etapa previa de glicerólisis (Fig. 1), empleando las mezclas de aceites, para lo cual se utilizó como catalizador hidróxido de sodio en una cantidad de $0.05 \%$ mol con respecto al aceite (Patton, 1962; Cardona, et al 2010). La relación molar glicerol/aceite fue 2.4 para todos los ensayos. Para determinar la composición de los productos obtenidos en la etapa de glicerólisis se utilizó un cromatógrafo de gases con detector FID y una columna DB-5HT de 15 metros; en la preparación de las muestras se utilizaron como agente derivatizante MSTFA (Sigma Aldrich), tetradecano (Merk) como estándar interno y como patrones de referencia (Sigma Aldrich) el gliceril palmitato, gliceril estearato, gliceril oleato y glicerol monoricinoleato. También se verificó una prueba cualitativa evaluando la solubilidad completa de producto en una relación 1:3(v/v) en metanol (Cardona, et al 2010).

\section{Etapa de policondensación:}

Las diferentes síntesis de las resinas se realizaron empleando una formulación basada en criterios de funcionalidad promedio (Patton, 1962). Inicialmente se adecuaron los aceites para el proceso de glicerólisis. El proceso de glicerólisis se llevó a cabo como se muestra en la

Fig. 2. Se fija una agitación mayor a $80 \mathrm{rpm}$, luego de alcanzar una temperatura de $160{ }^{\circ} \mathrm{C}$ se adiciona la glicerina, posteriormente se lleva a $190^{\circ} \mathrm{C}$ y se adiciona el catalizador, luego el sistema se calienta 
lentamente a $230{ }^{\circ} \mathrm{C}$ y se mantiene de 30 a 40 minutos, se toma una muestra y se determina la solubilidad en metanol ( $1 \mathrm{ml}$ de muestra en $3 \mathrm{ml}$ de metanol); si el sistema no solubiliza se debe mantener a $230^{\circ} \mathrm{C}$ por 15 minutos más y repetir la prueba; cuando sea soluble se procede a enfriar el sistema a $150{ }^{\circ} \mathrm{C}$ y se adicionan los otros reactivos: anhídrido ftálico, pentaeritritol, anhídrido maleico y etilenglicol, en las concentración mostradas en la Tabla 2.<smiles></smiles><smiles>CCC(C)(C)C(O)CO</smiles><smiles>[R]OC(=O)CC(O)CO</smiles>

Alfa-monoglicérido

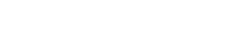<smiles>[R]OC(CO)CO</smiles>

Beta-monoglicerido<smiles>[R]O[R]([R])=O</smiles>

Alfa, beta-diglicérido Alfa, alfa-diglicérido

Fig. 1. Reacción de alcohólisis de aceites vegetales

Para llevar a cabo esta reacción se realizaron las siguientes mezclas de aceites de acuerdo a la Tabla 1.

Tabla 1: Porcentajes de mezclas de aceite de soja con otros aceites como palma, higuerilla y fritura.

\begin{tabular}{lrr}
\hline Ensayo & Aceite soja $\%$ & Otro aceite (\%) \\
\hline 1 & 0 & 100 (Higuerilla) \\
2 & 70 & 30 (Fritura) \\
3 & 70 & 30 (Higuerilla) \\
4 & 70 & 30 (Palma) \\
5 & 50 & 50 (Fritura) \\
6 & 50 & 50 (Palma) \\
7 & 50 & 50 (Higuerilla) \\
8 & 100 & 100 (Palma) \\
9 & 0 & 100 (Fritura) \\
10 & 0 & 70 (Fritura) \\
11 & 30 & 70 (Palma) \\
12 & 30 & 70 . (Higuerilla) \\
13 & 30 &
\end{tabular}

Tabla 2: Formulación de resinas alquídica

\begin{tabular}{lll}
\hline Materia prima & \% Peso & Estructura genérica de la resina alquídica \\
\hline Aceites & $20-25$ & $3-6$ \\
Etilenglicol & $20-22$ \\
Anhídrido ftálico & $7-12$ \\
Pentaeritritol & $0,5-2$ \\
Anhídrido Maleico & $2-5$ \\
Xilol & 40 & \\
Solvente de dilución Xilol & 0
\end{tabular}

Luego de cargar las materias primas se debe mantener a $150{ }^{\circ} \mathrm{C}$ hasta homogenización del sistema, se aumentan lentamente la temperatura hasta $220^{\circ} \mathrm{C}$ para que se dé la poli- condensación y periódicamente 
se chequea la acidez con el fin de conocer el estado del proceso, luego de que la resina está en una acidez menor de $10 \mathrm{mg} \mathrm{KOH} / \mathrm{g}$ muestra y presenta una viscosidad Gardner mayor de Z3, se procede al descargue de la resina lo cual se realiza en el solvente de dilución (xilol) y disminuyendo la temperatura; posterior al proceso de polimerización, la identificación y control de estos valores de acidez y viscosidad son la evidencia de la formación de un poliéster alquídico constituido fundamentalmente por cadenas que enlazan di-ácidos, polioles y los productos de la reacción de glicerólisis; en la Tabla 2 se presenta la estructura genérica de una resina alquídica con esta formulación.

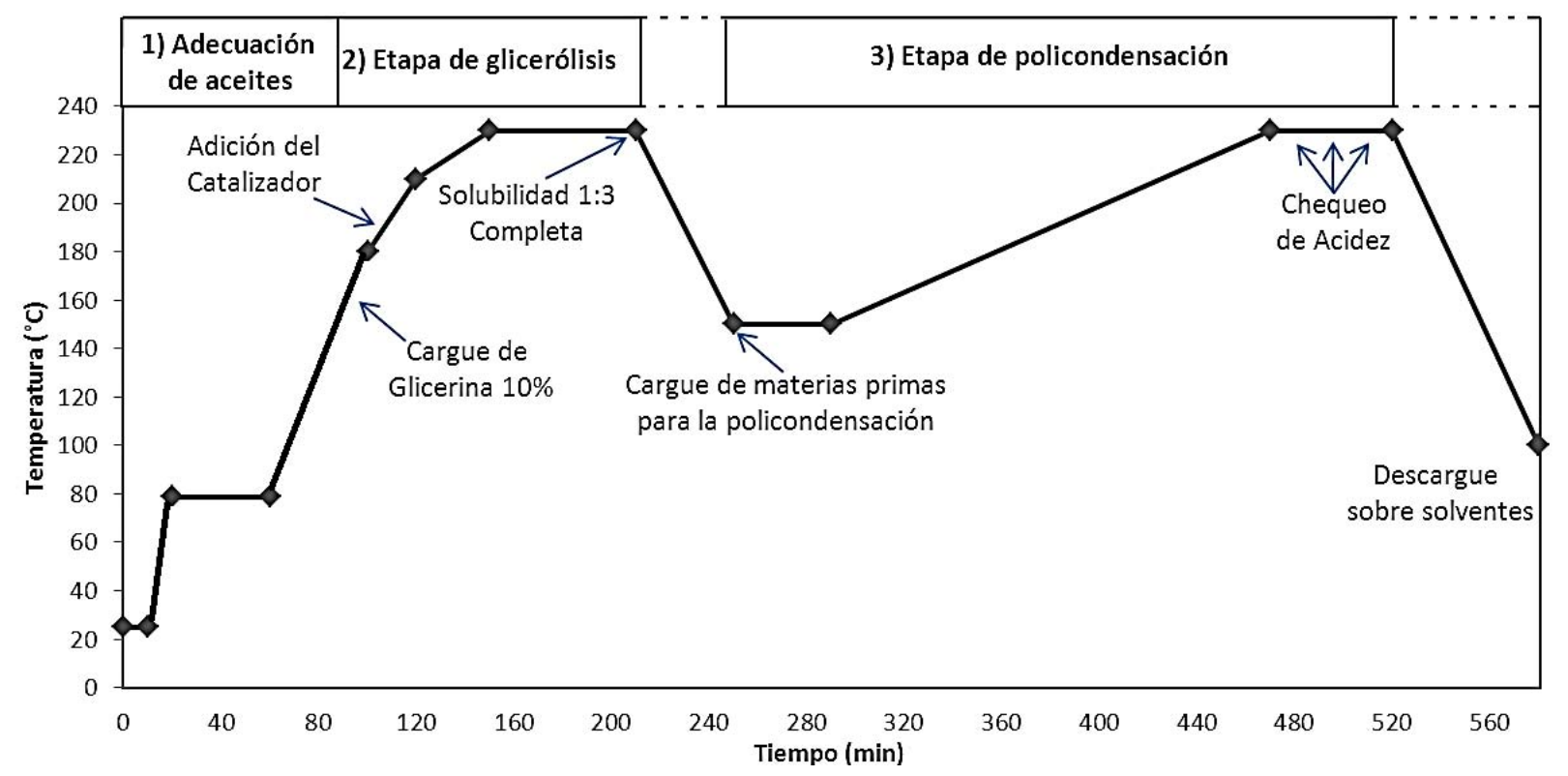

Fig. 2. Proceso general para la producción de una resina alquídica.

\section{RESULTADOS Y DISCUSIÓN}

\section{Caracterización fisicoquímica de los aceites vegetales}

Las características fisicoquímicas de los aceites crudos y de fritura empleando normas ASTM son presentadas en la Tabla 3. Así mismo se muestra la composición típica de cada aceite, expresado como perfil de metilésteres.

Tabla 3: Composición química y propiedades fisicoquímicas de aceites después de tratamiento.

\begin{tabular}{llrrrr} 
Índice & Norma aplicada & Fritura & Palma & Higuerilla & Soja RBD \\
\hline Yodo (g l/100 g muestra) & ASTM D5554 & 100,2 & 56,5 & 88,5 & 139,1 \\
Saponificación (mg KOH/g muestra) & ASTM D5558 & 191,40 & 198,3 & 181,5 & 193,3 \\
Acidez (mg KOH/g muestra) & ASTM D1980 & 1,65 & 0.35 & 0,53 & 1,02 \\
Hidroxilo (mg KOH/g muestra) & ASTM D1957 & n.a & n.a & 162.5 & n.a \\
Humedad (\% peso) & ASTM D4377 & 0,076 & 0.084 & 0,082 & 0.906 \\
& Láurico & 0,00 & 0,07 & 0,00 & 0,00 \\
& Mirístico & 0,54 & 0,83 & 0,00 & 0,08 \\
& Palmítico & 16,36 & 41,72 & 0,87 & 11,34 \\
& Palmitoléico & 0,85 & 0,13 & 0,00 & 0,08 \\
Composición química en términos de & Esteárico & 5,87 & 4,77 & 0,63 & 3,87 \\
ácidos grasos Método interno & Oléico & 37,45 & 41,31 & 4,09 & 22,63 \\
(cromatografía gaseosa) & Linoléico & 37,56 & 10,09 & 3,87 & 55,23 \\
& Linolénico & 0,86 & 0,90 & 0,45 & 6,77 \\
& Ricinoleico & 0,38 & 0,00 & 89,93 & 0,00 \\
& Eicosanoico & 0,05 & 0,07 & 0,06 & 0,00 \\
& Eicosenoico & 0,08 & 0,11 & 0,10 & 0,00 \\
& Saturados & 22,82 & 47,46 & 1,56 & 15,29 \\
\hline
\end{tabular}




\begin{tabular}{|c|c|c|c|c|c|}
\hline & Monoinsaturados & 38,38 & 41,55 & 94,12 & 22,71 \\
\hline & Poliinsaturados & 38,80 & 10,99 & 4,32 & 62,00 \\
\hline
\end{tabular}

Caracterización de las resinas alquídicas

Las propiedades fisicoquímicas de las resinas alquídicas obtenidas de las mezclas propuestas son presentadas en la Tabla 4.

Tabla 4: Propiedades fisicoquímicas finales de las resinas alquídicas

\begin{tabular}{|c|c|c|c|c|c|}
\hline & Ensayo $^{1}$ & $\begin{array}{r}\text { No volátiles. } \\
\text { (\%, ASTM D1644) }\end{array}$ & $\begin{array}{r}\text { Viscosidad } \\
\text { Gardner } \\
\text { (ASTM D1725) } \\
\end{array}$ & $\begin{array}{r}\text { Color } \\
\text { Gardner comparativo } \\
\text { (BYK TKD8-02) }\end{array}$ & $\begin{array}{r}\text { Índice } \\
\text { Acidez } \\
\text { (ASTM D1639) } \\
\end{array}$ \\
\hline 1 & & 57,01 & Q-R & 1 & 4,20 \\
\hline 2 & & 56,85 & Z9-Z10 & $7-8$ & 6,1 \\
\hline 3 & & 56,41 & Z2-Z3 & $5-6$ & 5,1 \\
\hline 4 & & 57,43 & Z2-Z3 & 6 & 7,8 \\
\hline 5 & & 57,55 & Z4-Z5 & $5-6$ & 5,2 \\
\hline 6 & & 55,26 & Z2 & $5-6$ & 2,8 \\
\hline 7 & & 57,81 & Z4 & 6 & 3,2 \\
\hline 8 & & 58,36 & Z4-Z5 & 3 & 4,4 \\
\hline 9 & & 54,09 & W-X & $5-6$ & 5,6 \\
\hline 10 & & 56,96 & Z-Z1 & $7-8$ & 7,0 \\
\hline 11 & & 57,25 & Z6-Z7 & $6-7$ & 0,71 \\
\hline 12 & & 57,03 & $\mathrm{Z1-Z2}$ & $5-6$ & 0,97 \\
\hline 13 & & 57,00 & Z6 & $4-5$ & 0,92 \\
\hline
\end{tabular}

En cuanto al porcentaje de volátiles, el valor recomendado para resinas alquídicas es de 57,0 $\pm 2,0 \%$, según los requerimientos de calidad de las normas técnicas NTC 1651. El porcentaje de volátiles de todos los ensayos de la tabla 4 cumple con este criterio de calidad.

Para recubrimientos alquídicos la viscosidad se establece según requerimientos de la norma NTC 996, que busca garantizar la calidad en la aplicación de recubrimientos (diluir hasta Y-Z1) tipo alquídicos (nivelación y chorreo); de esta manera los ensayos 1 y 9 tienen viscosidades muy bajas que nos son favorables para la aplicación.

El parámetro de color debe tener un valor no mayor a 3, en escala de color Gardner, para aplicaciones en lacas, barnices y esmaltes para acabados; mientras que valores más altos de color se pueden usar en preparadores de superficie como anticorrosivos para metales y selladores para madera. Este punto por lo general es establecido por criterios de calidad de las empresas y se referencia en el numeral 3.4. de la norma NTC 1651. Solo los ensayos 1 y 8 conducen a resinas aptas para lacas, barnices y esmaltes para acabados.

El rango de acidez se estableció de acuerdo a la experiencia propia y a la consulta de otros autores (Khurshid, 2006). El índice de acidez se mide con base en la resina solventada (no en base seca) y se recomiendan índices de acidez no mayores a 10. Los índices de acidez de todos los ensayos están por debajo de este valor.

De acuerdo a la tabla 6 y a los valores reportados en la literatura técnica (Valencia, 2005 y Khurshid, 2006) la mayoría de las resinas sintetizadas cumplen con los parámetros de calidad establecidos en la industria de las resinas alquídicas; se exceptúa el valor de la viscosidad del ensayo 1 que pueden ocasionar problemas de aplicación como chorreo y mala nivelación del recubrimiento debido a su baja viscosidad.

\section{Análisis Termogravimétrico (TGA)}

Se realizó análisis TGA sobre condiciones de aire atmosférico a una velocidad de calentamiento de $3^{\circ} \mathrm{C} / \mathrm{min}$ en un equipo LINSEIS STA Platinum Series. Los resultados de la Fig. 3 presentan pérdidas de peso mayores en la resina con $100 \%$ de aceite de soja y menores para las mezclas con los aceite de palma, fritura e higuerilla. Este comportamiento es esperado debido a que el aceite de soja $100 \%$ presenta mayor contenido de ácidos grasos poli-insaturados (Tabla 3) que las mezclas referidas en la Fig. 3. En general los aceites son más inestables térmicamente, a mayores contenidos de ácidos grasos poli-insaturaciones. En cuanto a la pérdida de peso de la mezcla con aceite de higuerilla se deberá tener en cuenta la influencia del 
grupo hidroxilo que tiene en su estructura; es conocido que el aceite de higuerilla $100 \%$ tiene mayor estabilidad térmica y oxidativa que los aceites de palma y soja (Zuleta, et al 2012); esto puede explicar la menor pérdida de peso de la resina que empleó la mezcla de aceite de higuerilla con soja

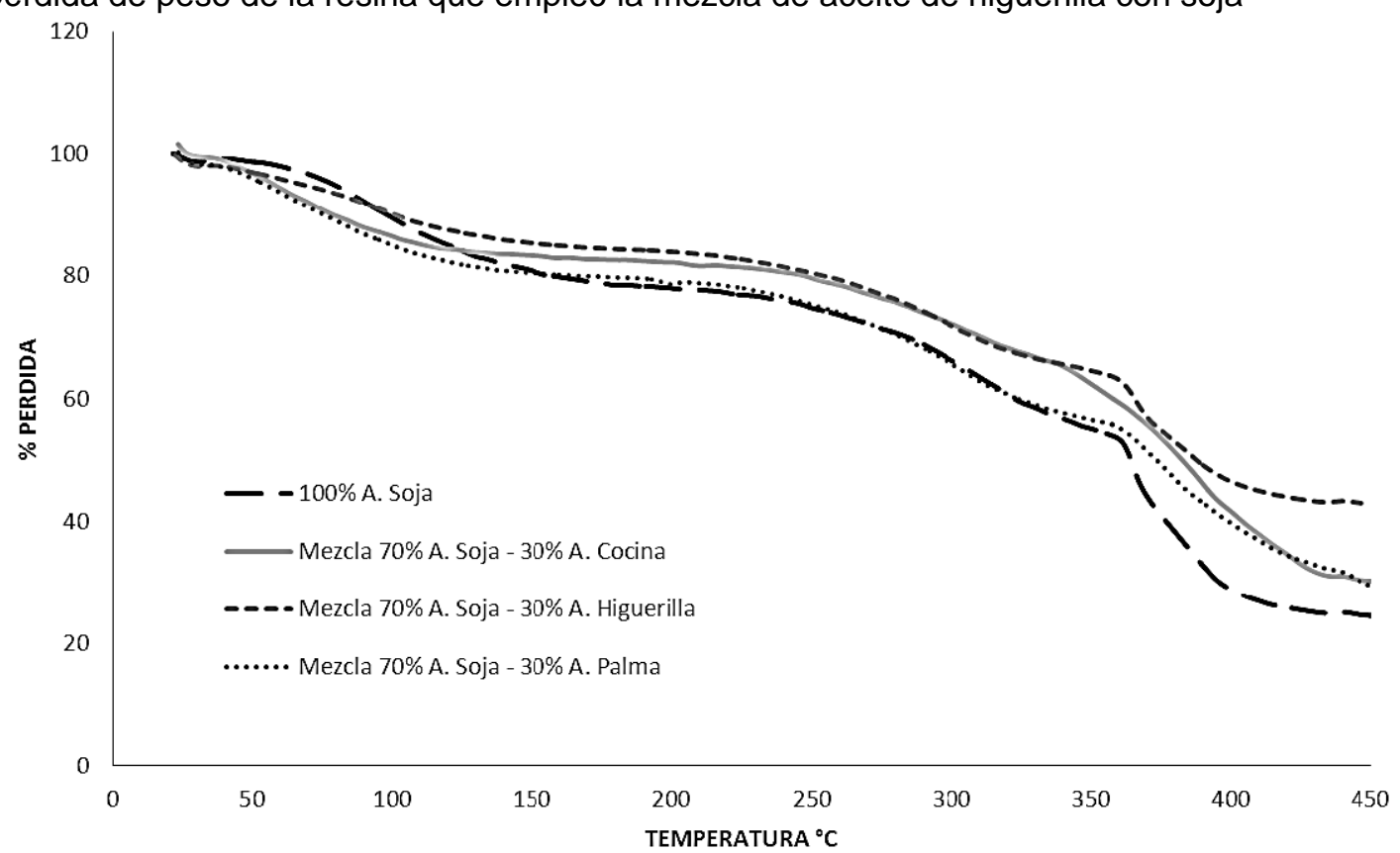

Fig. 3. Análisis TGA de resinas alquídicas con mezclas de aceite con soja al 70\%

Se concluye que las resinas fabricadas a partir de las mezclas de aceite de soja RBD y los aceites de palma, higuerilla y fritura tienen menores perdidas de peso y por lo tanto se esperaría una mayor estabilidad térmica comparadas con las resinas producidas con aceite de soja 100\%; pero será necesario evaluar un estudio más detallado con otros parámetros de calidad en estas resinas (mezclas del aceite de soja y otros aceites) para definir su viabilidad técnica.

\section{Análisis FT-IR}

En la Fig. 4 se presentan los espectros infrarrojo de las resinas alquídicas con sustitución total del aceite de soja RBD por los aceites de palma, higuerilla y fritura. Estos análisis sólo se muestran para este tipo de resinas y no para las de sustitución parcial, pues los cambios observados en estas últimas no son apreciables para su análisis. 


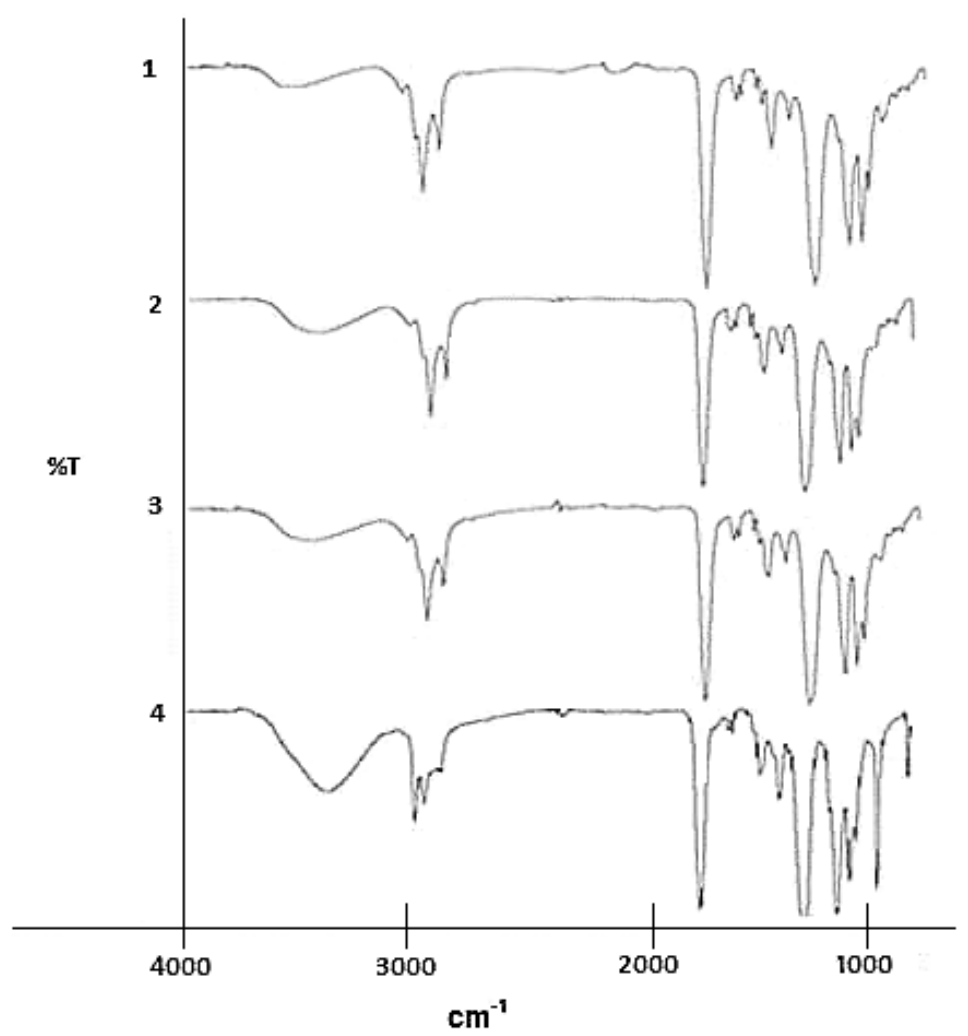

Fig. 4. Espectros FT-IR para las resinas alquídicas: (1) soja 100\%, (2) palma 100\%, (3) fritura 100\% y (4) higuerilla $100 \%$.

En el caso de la resina que empleó la mezcla con aceite de higuerilla, el grupo O-H secundario de la molécula en la cadena carbonada puede presentar mayor impedimento estérico que lo hace menos reactivo frente al di-ácido, esto ve reflejado en una intensidad mayor de la banda de los hidroxilos mostrada en el espectro $4\left(3380 \mathrm{~cm}^{-1}\right)$, en comparación con la de los espectros 1, 2 y 3 . De la misma forma los hidroxilos secundarios de los monoglicéridos y diglicéridos (véase figura 1) pueden tener el mismo comportamiento tal como se evidencia en los espectros 1,2 y 3 en la banda $3380 \mathrm{~cm}^{-1}$, las cuales tienen una menor intensidad que la resina con aceite de higuerilla.

\section{CONCLUSIONES}

Las resinas alquídicas obtenidas a partir de mezclas de aceite de palma, higuerilla y fritura con aceite de soja RBD (30\%-70\%, 50\%-50\%, 70\%-30\%, respectivamente) cumplen con los parámetros de calidad exigidos a las resinas poliméricas del tipo poliéster insaturado para su uso y aplicación como materias primas en la fabricación de recubrimientos de preparación de superficie y acabados. Por tanto se considera que el desarrollo es viable técnicamente para la sustitución parcial y/o total del aceite insaturado de soja por aceites de higuerilla, palma e incluso aceites de fritura en la fabricación de resinas alquídicas tipo poliéster para el desarrollo de recubrimientos de superficie.

Los resultados del análisis de TGA, FTIR y color Gardner muestran que los productos obtenidos contienen las estructuras químicas necesarias para llevar a cabo los procesos de curado oxidativo que requiere la resina e incluso algunas sustituciones favorecen la estabilidad oxidativa y la degradación de la película al someterla a condiciones extremas de temperatura. Estos resultados hacen interesante evaluar estas resinas para la fabricación de recubrimientos de alto desempeño como esmaltes horneables para sistemas especiales sometidos a altas temperaturas.

\section{AGRADECIMIENTOS}

Los autores agradecemos al COLCIENCIAS y a la Universidad de Antioquia (programa Estrategia de Sostenibilidad de Grupos) por su apoyo financiero.

\section{REFERENCIAS}

ASTM D5558-95: Standard Test Method for Determination of the Saponification Value of Fats and Oils. West Conshohocken, PA, United States (2011). 
ASTM D5554: Standard test method for determination of the iodine value of fats and oils. West Conshohocken, PA, United States (2011).

ASTM D1980-87: Standard Test Method for Acid Value of Fatty Acids and Polymerized Fatty Acids. West Conshohocken, PA, United States (1998).

ASTM D1957-86: Standard Test Method for Hydroxyl Value of Fatty Oils and Acids. West Conshohocken, PA, United States (2001).

ASTM D4377-11: Standard Test Method for Water in Crude Oils by Potentiometric Karl Fischer Titration. West Conshohocken, PA, United States (2011).

ASTM D1644-12: Standard Test Methods for Nonvolatile Content of Varnishes. West Conshohocken, PA, United States (2012).

ASTM D1725-12: Standard Practice for Preparing Resin Solutions for Viscosity Measurement by Bubble Time Method. West Conshohocken, PA, United States (2012).

ASTM D1639-90: Standard Test Method for Acid Value of Organic Coating Materials. West Conshohocken, PA, United States (1990).

Bakker, P., J. Baltusen y M. Bolin, Emulsification Strategy of Alkyd Resins. Russian Coatings Scientific association, 1, 1-12 (2004).

Cardona, S.M. y otros cuatro autores, Production of monoglycerides from castor oil using crude and refined glycerin: Study of the main variables of the process, Alimentos: Ciencia, Tecnología E Ingeniería, ISSN 0121-4004 (2010-en línea),

Dullius, J. y otros cuatro autores, Chemical recycling of post-consumer PET: Alkyd resins synthesis. Progress in Organic Coatings 57, 123-127 (2006).

Hlaing, N.N. y M.M. Oo, Manufacture of alkyd resin from castor oil. Proceedings of World Academy of Science, Engineering and Technology: 36, 928-934, (2008).

Isaam, A.M. y C.Y. Cheun, A study of the effect of Palm Oil on the properties of a new Alkyd Resin. Malaysian Polymer Journal, 4(1), 42-49 (2009).

Jinkyung K., y otros cinco autores, Synthesis and applications of unsaturated polyester resins based on PET waste, Korean Journal of Chemical Engineering: 24(6), 1076-1083 (2007).

Karayannidis, G.P., y otros tres autores, Alkyd resins derived from glycolized waste poly(ethylene terephthalate). European Polymer Journal, 41, 201-210 (2005).

Khurshid, M., Production of alkyd resin. Dhahran: Summer training report, King Fadh University of petroleum and minerals. (2006)

Lafont, Jennifer y otros dos autores, Análisis Químico de Mezclas Biodiésel de Aceite de Cocina Usado y Diésel por Espectroscopía Infrarroja. Información Tecnológica, vol.22, no.4, p.35-42. (2011)

Lapuerta, M., R. Fernández, y J, Agudelo, Diesel particulate emissions from used cooking oil biodiesel, Bioresource Technology: 99, 731-740 (2008).

Marrion A., Film formation. The Chemistry and Physics of Coatings, Segunda edición, Published by The Royal Society of Chemistry, 49-59, Northampton, UK, (2004).

Murillo, E.A. y B.L. López., Novel waterborne hyperbranched acrylated-maleinized alkyd resins. Progress in Organic Coatings: 72, 731- 738 (2011)

NTC 996: Pinturas y productos afines. Aplicación en láminas de ensayo, Bogotá DC, Colombia (1975).

NTC 1651: Pinturas. Imprimantes anticorrosivos con vehículo alquídico, Bogotá DC, Colombia (1997).

Panda, H., Alkyd Resins Technology Handbook. Asia Pacific Business Press Inc, 1, New Delhi, India (2010). 
Patton, T.C., Alkyd resin technology, formulating techniques and allied calculations. Manual \# 8. New York: Interscience: 197, (1962).

Quiñonez, L., Dimensionamiento y Montaje de un Reactor Piloto Para la Obtención de Biodiesel a partir de Aceites Vegetales Gastados. Tesis de grado ingeniero químico, Universidad Nacional de Colombia UNAL sede Bogotá, Facultad de Ingeniería, (2003).

Sevim, Z.E., L. Zengshe y X. Jingyuan, Preparation characterization and mechanical properties of epoxidized soybean oil/clay nanocomposites, Polymer: 46(23), 10119-10127, (2005).

Torlakoglu, A. y G. Guclu, Alkyd-amino resins based on waste PET for coating applications, Waste Management: 29, 350-354. (2009)

Valencia, S.A., Memorias "Seminario sobre Polimerización, Reactores y Aceros Inoxidables, Seminario ofrecido por la Sociedad de Ingenieros Químicos de la UPB. Medellín, Colombia, septiembre 19 a octubre 7 de (2005).

Wallace, J.G., Encyclopedia of Chemical Technology, 3rd ed., john Wiley \& Sons, Vol. 9, pp 795-830, New York (1978).

Zuleta, E.C., L.A. Rios y P.N. Benjumea, Oxidative stability and cold flow behavior of palm, sacha-inchi, jatropha and castor oil biodiesel blends. Fuel Processing Technology 102, 96-101 (2012). 
